\title{
AN EVALUATION OF A CONSERVATIVE TRANSMIT POWER CONTROL MECHANISM ON AN INDOOR 802.11 WIRELESS MESH TESTBED
}

\author{
Karol Kowalik, Marek Bykowski, Brian Keegan and Mark Davis \\ Communications Network Research Institute, Dublin Institute of Technology, IRELAND \\ karol.kowalik@cnri.dit.ie,marek.bykowski@cnri.dit.ie,brian@cnri.dit.ie,mark.davis@dit.ie
}

Keywords: $\quad$ Transmit Power Control, Wireless Mesh, 802.11.

Abstract: $\quad$ Power control techniques for IEEE 802.11 wireless networks have already gained considerable attention. Such techniques are particularly attractive because they can improve various aspects of wireless network operation such as interference mitigation, spatial reuse in dense wireless deployments, topology control, and link quality enhancement. In this paper we propose a novel delivery ratio based Conservative Transmit Power Control (ConTPC) mechanism. Our implementation is conservative when it comes to deciding if the transmit power should be reduced for a given link. This is because we do not want poor quality wireless links to further reduce their quality and be overwhelmed by other links transmitting at maximum power. We have experimentally evaluated the benefit of the proposed power control scheme when compared with fixed power level systems. We show that our ConTPC mechanism can increase the throughput, however the magnitude of this enhancement largely depends on the topology of the wireless network.

\section{INTRODUCTION}

Interference has been identified as a key cause of performance degradation in Wireless Mesh Networks (WMNs) (Jain et al., 2003; Padhye et al., 2005). Transmit power control is one of the methods which allows for interference mitigation and spatial reuse through per-link power control.

Per-link power control allows a network node to transmit packets to its neighbours at different power levels. Data transfers to close neighbours may be performed at low transmission powers, thereby minimising the interference with remote nodes. Conversely, the communication with remote neighbours may be improved by using a higher transmission power, i.e. by providing a stronger signal at the receiver. It has been shown by Muqattash et al (Muqattash and Krunz, 2005) that a power controlled MAC (POWMAC) protocol can improve significantly improve network throughput. Furthermore, power control becomes a necessity in dense deployments which are now becoming common as demonstrated by Akella et al. (Akella et al., 2005).

However, increasing the transmission power for weak links also has a negative effect of producing increased interference. There are other factors which can impact on the benefit of employing transmit power control. For example, Broustis et al. (Broustis et al., 2007) have observed that when power control is combined with virtual carrier sensing (RTS/CTS messages) the performance is often degraded.

Transmit Power Control (TPC) mechanisms when implemented in WMNs can be used to:

- minimise interference with other nodes (and thus increasing spatial reuse) as implemented in POWMAC (Muqattash and Krunz, 2005) or in (Navda et al., 2007);

- improve the quality of wireless links (as implemented in (Son et al., 2004));

- reduce the energy consumption (Gomez et al., 2003);

- control the network topology (Ramanathan and Hain, 2000);

- reduce interference with satellites and radar operating in the $5 \mathrm{GHz}$ frequency band (as required by the IEEE 802.11h Standard (802.11h IEEE Standard for Information technology - Telecommuni- 
cations and information exchange between systems - Local and metropolitan area networks Specific requirements, ));

- ensure good coverage (as implemented by some access point manufacturers).

Some of these objectives can be realised by modifying a single layer, such as the POWMAC (Muqattash and Krunz, 2005). However, in general power control needs to be aware of the operation at multiple layers and is more accurately a cross-layer optimisation problem. Kawadia and Kumar present an comprehensive discussion about the principles of such a cross layer design of power control in (Kawadia and Kumar, 2005) .

The majority of the power control techniques proposed require power control with per-packet level granularity and with low latency. In (Kowalik et al., 2008) we have shown that WLAN cards with Atheros chipsets allow for such a fine power control.

In this paper we present a novel delivery ratio based Conservative Transmit Power Control (ConTPC) mechanism. We evaluate it experimentally on an eleven node static indoor 802.11 wireless mesh testbed as well as on a four node topology which provides an easy to analyse example of a disjoint communication case.

\section{RELATED WORK}

Reports in (Mon, 2001; Muqattash and Krunz, 2003) present 802.11 MAC modifications which aim to utilise power control. However, these solutions require the use of a separate control channel for the exchange of collision avoidance information. Such schemes would require two wireless interfaces at each mesh node. Thus this approach is not feasible for the single radio solution considered in this work.

The POWMAC (Muqattash and Krunz, 2005) proposed by Muqattash and Krunz is an enhancement of the 802.11 MAC enabling power control and resembles the ConTPC scheme proposed in this paper. However, POWMAC requires detailed information about the power of a received control signal, as well as the interference power. This may not be a problem if one uses simulation tools to evaluate the approach as the authors did. However, this is unrealistic because we and other researchers have observed that SNR measurements exhibit a high variability. For example Akella et al. (Akella et al., 2005) have shown that $4 \mathrm{~dB}$ of variance in RSSI values and noise estimates is typical. Moreover, under the POWMAC scheme the transmission of each data packet is preceded by an access window (AW) during which several pairs of neighboring terminals exchange their RTS/CTS control messages. Consequently this can generate a significant overhead. There is a similarity between POWMAC and LPERF (Akella et al., 2005) (Loadsensitive, Power-controlled Estimated Rate Fallback) because both combine the load information with signal strength measurements. However, it was stated by Akella et al. (Akella et al., 2005) that in practice they found that "achieving good performance and interference reduction using the LPERF technique can be challenging" owing to the poor accuracy of signal strength measurements and the difficulties in estimating the load.

Broustis et al. (Broustis et al., 2007) performed physical experiments and have analysed three scenarios in which power control may improve network performance:

- "overlapping case" - where power control cannot improve the performance of two overlapping links;

- "hidden terminal case" - where power control can improve fairness;

- "potentially disjoint case" - where power control can improve throughput significantly.

Their experiments show that power control may improve overall throughput, however virtual carrier sensing (RTS/CTS messages) needs to be disabled. Also, their results demonstrate that the benefit from using power control is topology dependent. In Section 6.2 we also present similar findings.

Akella et al. (Akella et al., 2005) have proposed a number of combined power and rate control mechanisms. The one which resulted in the best performance was PERF (Power-controlled Estimated Rate Fallback) (Akella et al., 2005) which implemented conservative power control. PERF performs its decisions regarding the rate and transmit power based on the delivery rate of previous packets and SNR estimates obtained from the WLAN adapter. Moreover, each packet is extended to include information about the transmit power, path loss, noise estimate of the last packet, and delivery rate information. ConTPC adopts a similar approach. However, PERF reduces the transmit power until estimatedSNR $=$ decisionThreshold + powerMargin. The powerMargin is used to control aggressiveness of the algorithm. Our ConTPC scheme is also conservative, but it takes a different approach in trying to reduce power provided that it does not result in an increased packet loss. 


\section{CONSERVATIVE TRANSMIT POWER CONTROL (CONTPC)}

We believe that many of the proposed power control schemes are too opportunistic. The rationale behind these schemes is that by tolerating a degree of interference at each node, one can trade-off an increased BER (Bit Error Rate) for multiple simultaneous transmissions. For example, POWMAC (Muqattash and Krunz, 2005) follows this approach.

We have discovered that in real indoor deployments this approach may be overly optimistic and can degrade the network performance. In our wireless eleven node static indoor testbed wireless links often exhibit high loss rates. Moreover, their delivery rate vs TX power characteristics often resembles that shown in Figure 1. This sort of characteristic has the distinct feature that even a small reduction in the transmit power from its maximum value can result in a significant drop in the delivery rate. Moreover, when multiple simultaneous transmissions occur, the noise floor increases. Thus for a link with such a characteristic its delivery rate would drop even further. Experimentally we have found that for links with such a characteristic even small decreases in the transmit power can significantly degrade their performance.

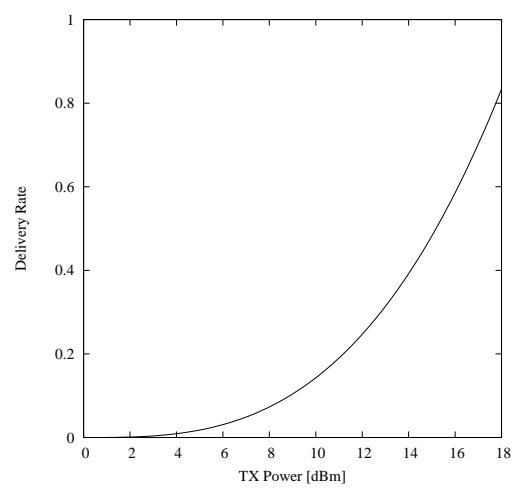

Figure 1: Delivery ratio vs. TX power characteristics for a low quality link

In our ConTPC mechanism we do not allow the power control mechanism to reduce the transmit power for all the network links. Instead the ConTPC mechanism first checks if the delivery rate vs TX power curve is flat around its maximum power level. For example, the characteristic could look like the one shown in Figure 2. On such a link any reduction in the TX power does not adversely impact on its delivery rate. Therefore, reducing the transmit power will typically not degrade the performance of the link, but can potentially reduce the interference on nearby links.

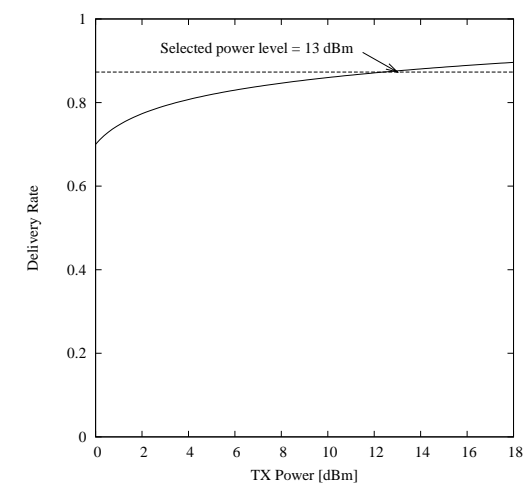

Figure 2: Delivery ratio vs. TX power characteristics for a high quality link

However, even for high quality links we do not advocate large reductions in the transmit power as this may expose the node to the risk of being overwhelmed by neighboring nodes transmitting at maximum power. Thus, our power control scheme adopts a quite conservative approach when it comes to reducing the transmit power on any given link. ConTPC will only reduce the transmit power while it does not adversely impact of the delivery rate. The exact details of our scheme are provided in the following section.

\section{CONTPC IMPLEMENTATION DETAILS}

Our ConTPC mechanism controls the output power on a per-link basis. Therefore, each node periodically broadcasts probe frames at all allowed power levels which includes information about the power level used to sent a given frame. This information allows each node to create delivery rate vs TX power characteristics for all its incoming links. In our experiments we have used custom probe messages, but HELLO messages which are usually exchanged between routers could also serve this purpose.

Our conservative approach requires the delivery rate vs TX power characteristic to be almost flat around the maximum power level. In order to describe the details of ConTPC power selection algorithm we denote:

$P_{\max }$ to be the maximum TX power level (for our WLAN adapters it was $18 \mathrm{dBm}$ ); 
$P_{\text {selected }}$ to be the power level selected by our ConTPC mechanism;

$P_{\text {step }}$ to be the granularity of our power mechanism;

$P_{\text {safety }}$ to be the safety margin, which defines the minimum range of the flat area around the maximum power;

thr to be the threshold value which defines the end of the flat area around the maximum power;

$d l r$ to be the delivery rate vs TX power characteristic.

In Figure 3 we present a diagram showing the details of ConTPC. Essentially, it selects the power level at the leftmost part of the flat region around the maximum TX power, provided that the size of this region is larger than the safety margin. ConTPC uses three configurable parameters: $P_{\text {step }}, P_{\text {safety }}$ and $t h r$. The $P_{\text {step }}$ together with the frequency of the power broadcasts can be used to tune the overhead and the accuracy of the estimation of the delivery rate characteristics. The $P_{\text {safety }}$ and $t h r$ parameters are used to control the aggressiveness of ConTPC. An evaluation of the relative importance of these parameters will be given in Sections 6.5 and 6.6.

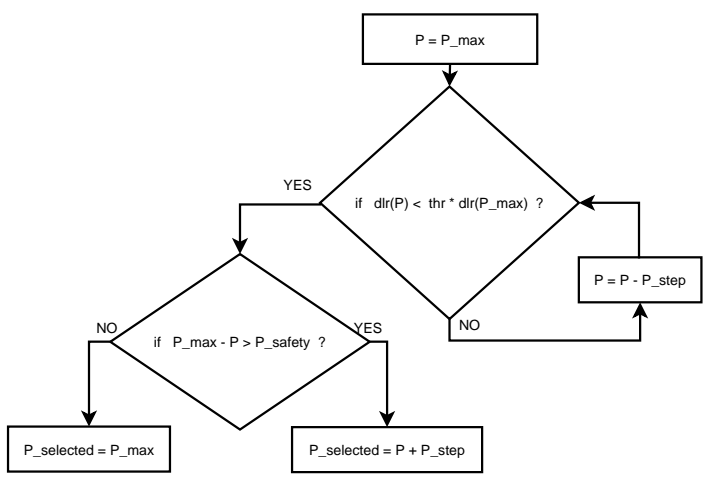

Figure 3: ConTPC - power selection details

The ConTPC mechanism allows a node to determine the transmit power levels for its incoming links. Once this has been done, each node needs to inform its neighbours of the transmit power level that they need to use when communicating with the node. In static wireless mesh networks the communication pattern usually resembles a tree whose main root is located at the gateway node. To reduce the overhead involved in informing neighbours about the power levels that they need to use, we propose to distribute this information along this tree-like communication pattern only. Consequently, power control is only performed on links within this communication pattern.

\section{EXPERIMENTAL SETUP}

We have implemented ConTPC as a Click (Kohler et al., 2000) module. This module allows us to broadcast custom made power probes, generate delivery rate vs TX power characteristics and inform neighbouring nodes about the power levels that they need to use.

To perform the experimental tests we used eleven WLAN nodes distributed across two floors of our research institute building as shown in Figure 4. The nodes were based around the Soekris net4521 platform. The experiments were performed indoors, and unfortunately we could not completely isolate the tested network from external sources of interference. All nodes were running Linux operating systems and were operating in the monitor mode to allow for packet injection by our Click module.

In order to enable the TPC feature of MadWifi drivers one needs to compile it with $\mathrm{COPTS}+=-\mathrm{DATH} \_\mathrm{CAP} \_\mathrm{TPC}=1$ and pass the tpc=1 parameter to insmod ath_pci.

Moreover, the transmission rate was fixed for all the experiments at $11 \mathrm{Mbps}$ and the antenna diversity was also switched off.

\section{RESULTS}

\subsection{Delivery ratio vs SNR}

In the ConTPC scheme each node measures the delivery ratio vs TX power characteristics of its incoming wireless links. In Figure 5 we have presented these characteristics for all links of our test bed. One might think that instead of the delivery ratio it would be easier to use Signal-to-Noise Ratio (SNR), since it directly relates to the power level used for the transmission. Moreover, SNR value can be easily obtained from the wireless card, for example Atheros chipsets report RSSI value which can by simply converted to SNR by subtracting $95 \mathrm{~dB}$.

However, we do not advocate the use of SNR because we have observed a poor accuracy on the RSSI measurements especially for weak signals as illustrated in Figure 6. Akella et al. (Akella et al., 2005) have shown that that the variance in RSSI estimates is high and is typically around $4 \mathrm{~dB}$. Moreover, in Figure 6 one may observe that the SNR vs TX power 


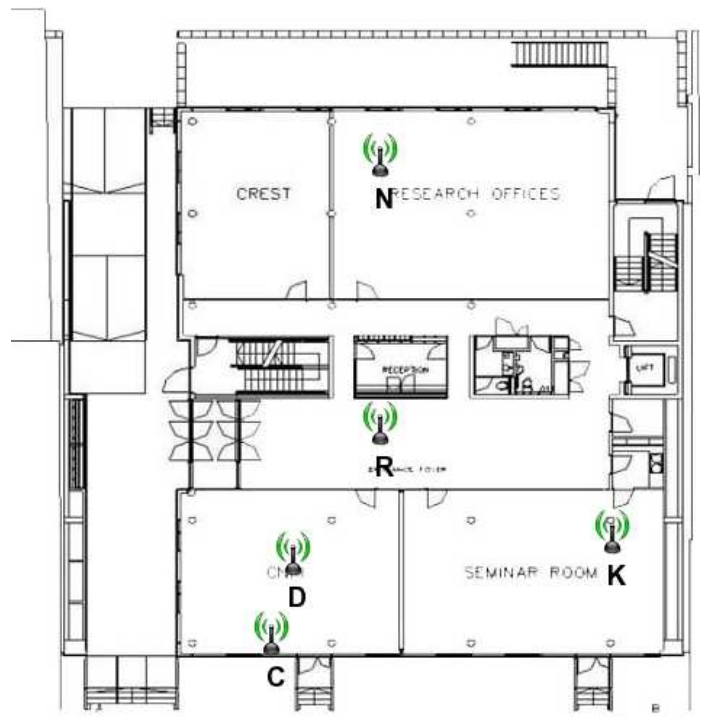

(a) Floor 1

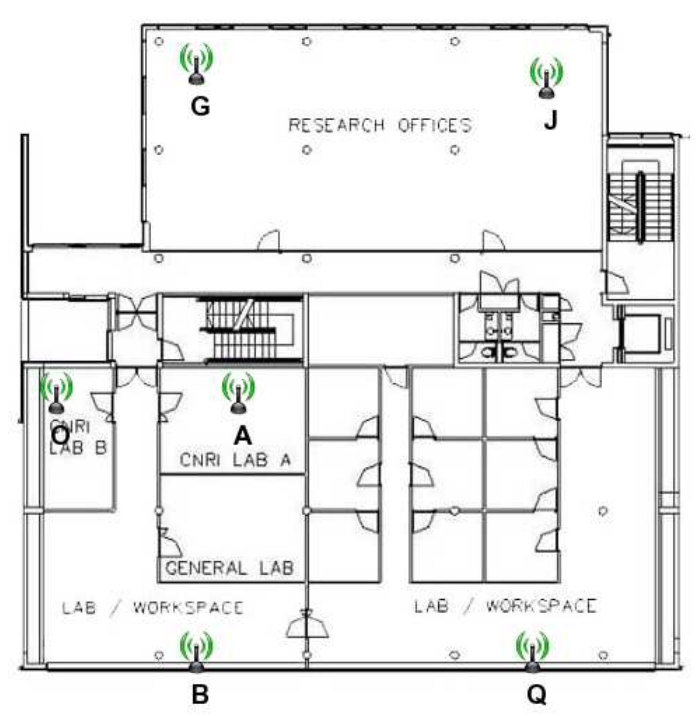

(b) Floor 2

Figure 4: Locations of wireless nodes

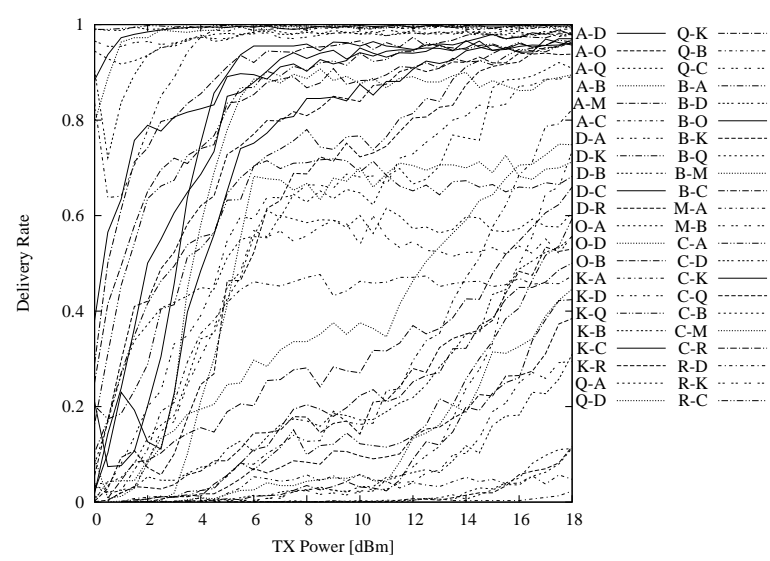

Figure 5: Delivery rate vs TX power

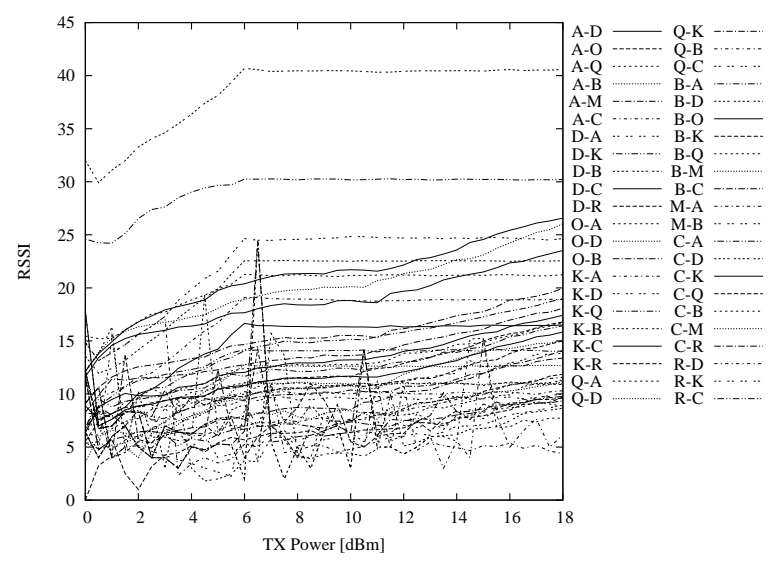

Figure 6: SNR vs TX power

\subsection{Disjoint case}

characteristics are much flatter than the delivery rate vs TX power curves.

In Figure 7 we show how the delivery rate is related to the RSSI values. Although, it can seen that there is a correlation between the SNR and the delivery rate, the large variance associated with the SNR measurements make it difficult to use. Consequently, in ConTPC we have decided to use the delivery rate instead of the SNR.
In order to validate the operation of the ConTPC scheme, we first decided to investigate how it performs in the disjoint case where the expected benefit is the greatest according to Broustis et al. (Broustis et al., 2007). In this case transmit power control can allow for the simultaneous use of two links which results in a twofold increase in throughput compared to the case where the default maximum transmit power is used. In general, if we were to have $n$ such node pairs all interfering with each other under maximum transmission power conditions, then we could expect 


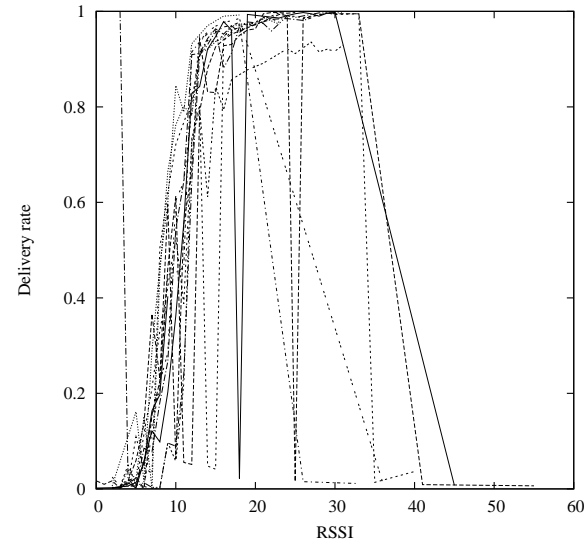

Figure 7: Delivery rate vs TX power

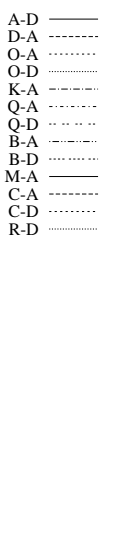

a $n$-fold increase in throughput through the use of power control. However, such topologies based on pairs of nodes located close to each other and traffic patterns based on communication between pairs of nodes only are quite rare.

In our testbed shown in Figure 4 we have moved node $\mathrm{B}$ towards $\mathrm{A}$ and $\mathrm{D}$ towards $\mathrm{B}$, to create a scenario with two disjoint pairs $\mathrm{A}-\mathrm{B}$ and $\mathrm{C}-\mathrm{D}$. Then we performed an experiment in which each node sent a $512 \mathrm{kbps}$ UDP flow to its neighbouring node. Thus we had four flows in the network and the experiment duration was 6 hours. During this period we have compared the performance of ConTPC with fixed power levels at $0 \mathrm{dBm}$ and at $18 \mathrm{dBm}$. To ensure that each of these power schemes is tested under similar conditions, they were dealt with in a round robin fashion over a duration of 1 min each.

As can be observed in Figure 8 the delivery rate characteristics were excellent for all power levels between pairs of nodes. Even though the node pairs were located on different floors the delivery rate on link B-D was still good, and also on links A-D and $\mathrm{C}-\mathrm{A}$. Therefore, these pairs were not completely disjoint, so contention on the link B-D was still high even though the minimum power of $0 \mathrm{dBm}$ was used. Thus we did not observe doubling of the throughput, as one might expect.

In Figure 9 we show that ConTPC resulted in an increase of $35 \%$ when compared with transmission at the maximum power of $18 \mathrm{dBm}$. What is also interesting here is that ConTPC slightly outperformed transmission at maximum power of $0 \mathrm{dBm}$. This is because it emerged that for links $\mathrm{B}-\mathrm{A}$ and $\mathrm{C}-\mathrm{D}$ it was more benefitial to use a transmit power of around 2 or $3 \mathrm{dBm}$ instead of the minimum value.

The nodes in our test bed are usually not placed in

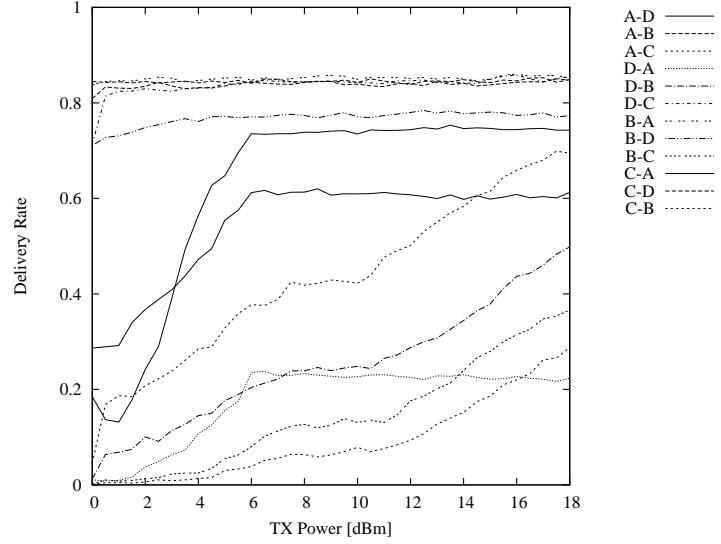

Figure 8: Delivery rate characteristics observed in disjoint case

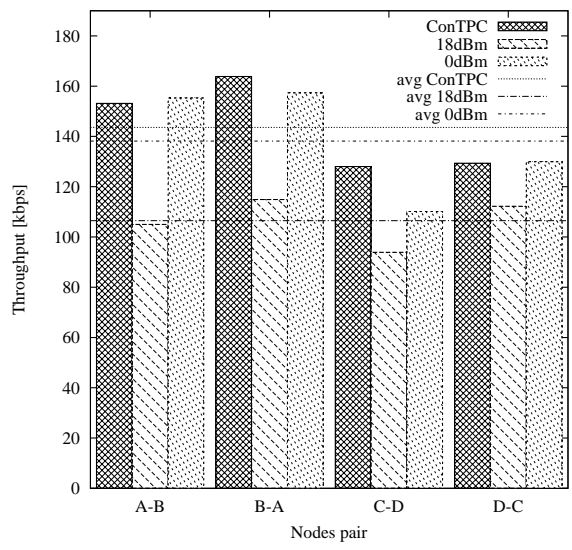

Figure 9: Throughput observed in disjoint case

pairs so close to each other, as presented in the above experiment. Therefore, for the next step in the investigation we moved the nodes back to their normal positions and repeated the tests. Thus, it was no longer disjoint case. As it can be seen in Figure 10 only link B-A continued to exhibit an excellent delivery rate characteristic. Thus, it achieved the highest throughput when a fixed power of $0 \mathrm{dBm}$ was used (as it is shown in Figure 11).

In Figure 11 it may be observed that in this more realistic 4 nodes case our ConTPC also outperforms fixed power levels at $18 \mathrm{dBm}$ and at $0 \mathrm{dBm}$. We also demonstrate that in such a case where nodes are distributed evenly around the area (usually to maximise coverage) the use of maximum power is close to optimal. Thus if the nodes were to be spread more uniformly to maximise coverage then we would observe that ConTPC would provide the same overall through- 


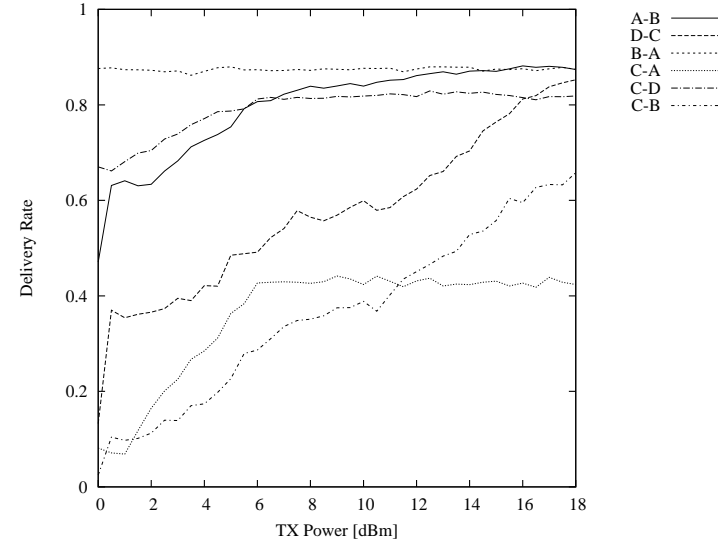

Figure 10: Delivery rate characteristics observed in 4 nodes case

put as when the maximum power of $18 \mathrm{dBm}$ is used.

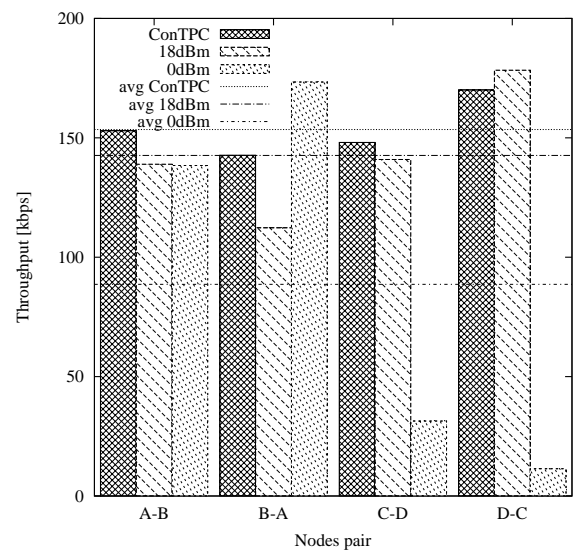

Figure 11: Throughput observed in 4 nodes case

This experiment demonstrates that the benefit of using transmit power control is topology dependent. It also shows that our ConTPC outperforms fixed power levels if there is some nonuniformity in the node distribution. Thus it can reduce the transmit power for strong links, thereby reducing the interference.

\subsection{Eleven nodes testbed case}

To evaluate ConTPC over our eleven node test bed we have used a similar procedure. However, this time the communication pattern was not performed in disjoint pairs. Instead each node was sending a 512kbps UDP flow to the node which was the next hop towards the gateway node. Such communication pattern is similar to the typical case when nodes want to connect to the Internet through a gateway node. We have decided not to forward the data packets beyond the gateway node as we wished to observe the effect of transmit power control on individual links instead of observing the effect of hop penalty. The duration of this experiment was 24 hours and all the power schemes were tested in a round robin fashion.

In Figure 12 we have compared the throughput achieved by the individual links on our eleven node testbed. It can be observed that the ConTPC mechanism resulted in approximately a 50\% increase in throughput when compared with a fixed power level at $0 \mathrm{dBm}$ and a $15 \%$ increase in throughput when compared with a maximum power of $18 \mathrm{dBm}$. One can

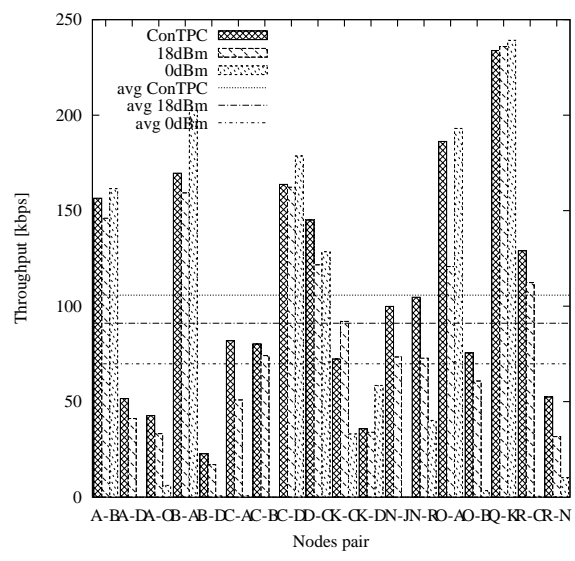

Figure 12: Throughput observed on 11 node testbed

also observe in Figure 12 that ConTPC offered an improvement mostly for links which achieved low or average throughput when the maximum power was used. This is because when the transmission was occurring at maximum power, these links were suffering due to increased contention and noise level. Reducing the transmit power of high quality links resulted in improved performance of weak links. So ConTPC has not only increased the average throughput, but has also increased the fairness.

\subsection{Stability of ConTPC}

In Figure 13 we present the probability distribution functions (PDFs) of the power level selection performed by ConTPC during the experiment performed in Section 6.3. This diagram allows one to determine how often ConTPC changes the power levels or if the power level remains fixed for extended periods of time. As can be observed on link A-B, ConTPC selected a wide range of power levels. This is because 

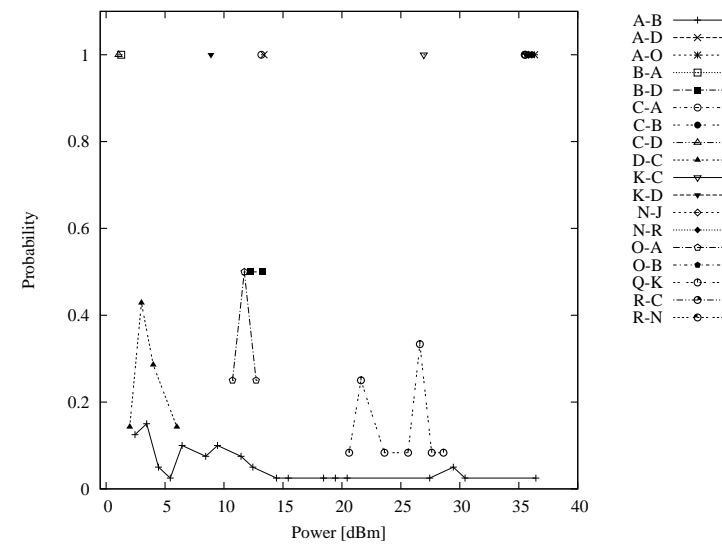

Figure 13: Power level selection PDF for links of 11 node testbed

node A was located in a corridor where there was a high variability in the propagation conditions due to the movement of people. However, most of the time this link exhibited a high delivery rate, thus the selected power tended to switch between 8 and $9 \mathrm{dBm}$.

For the majority of the links, the transmit power level was selected around a small range of values or remained at one value for the duration of the experiment.

\subsection{Overhead of ConTPC}

ConTPC obtains the delivery rate vs TX power characteristics by broadcasting power probes at all specified power levels. In this section we consider the direct overhead which is the amount of time consumed by the transmission of these broadcast probe packets.

The overhead can be controlled by the frequency of these broadcasts. In our experiment we have measured the overhead on node D which was the gateway node and featured the highest number of neighbours, and thus was influenced by the highest overhead. In Figure 14 we have presented the overhead as a percentage for three different intervals between consecutive broadcasts, i.e. every $50 \mathrm{~ms}, 100 \mathrm{~ms}$, and $200 \mathrm{~ms}$. As expected reducing the frequency of these broadcasts resulted in a drop in the overhead from $2.5 \%$ to $1.3 \%$, and finally to $0.6 \%$. Although the $200 \mathrm{~ms}$ interval resulted in the smallest overhead we have observed that ConTPC has significantly slowed the process of generation of the delivery rate vs TX power characteristics. Therefore, if one would wished to keep the overhead low at around $0.6 \%$, we would recommend to reduce the number of power levels being tested. In (Kowalik et al., 2008) we have shown that WLAN cards with Atheros chipsets allow for the con-

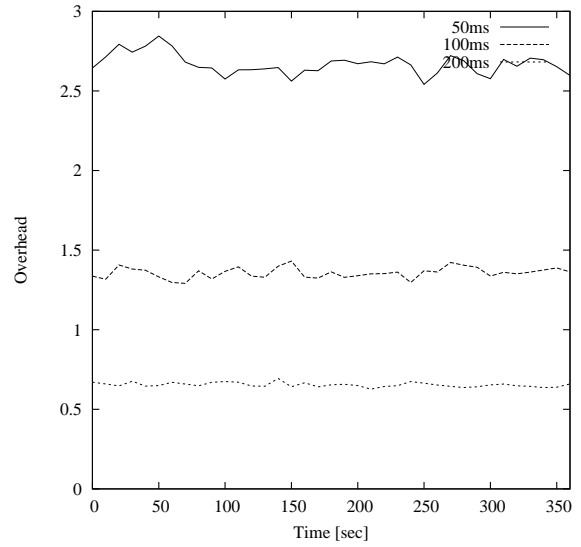

Figure 14: ConTPC overhead

trol of the transmit power with $0.5 \mathrm{dBm}$ granularity. Not all the power levels need to be used by the power control mechanism, especially if we wish to limit the probing overhead. Thus, for a $200 \mathrm{~ms}$ broadcast interval we have increased the value of $P_{\text {step }}$ form $0.5 \mathrm{dBm}$ to $1 \mathrm{dBm}$. The increase of $P_{\text {step }}$ with the broadcast interval set at $200 \mathrm{~ms}$ allowed ConTPC to continue to outperform tansmissions at maximum power for our eleven node test bed.

\subsection{ConTPC parameters evaluation}

ConTPC uses two configurable parameters $P_{\text {safety }}$ and $t h r$ to control its aggressiveness. The $P_{\text {safety }}$ is a safety margin, which defines the minimum range of the flat region around the maximum power. The $t h r$ is the threshold value which defines if the region is still flat around the the maximum power. Thus, if one would set $P_{\text {safety }}$ to be $0 \mathrm{dBm}$, and the $t h r$ to be equal to 0.9 , then all network links would use a transmit power which results in the delivery rate of $0.9 * d l r(18 d \mathrm{Bm})$. These settings would effectively reduce the transmit power on all the network links.

In Figure 15 we have provide evidence that the opportunistic approach where $\left(P_{\text {safety }}=0 \mathrm{dBm}\right)$ results in the worst performance. The conservative approach ( where $P_{\text {safety }}>=1 \mathrm{dBm}$ ) is much better. It can be observed that the highest average throughput is achieved if we select a low threshold $t h r=0.8$ and use a wide power safety margin as $P_{\text {safety }}=4 \mathrm{dBm}$. Setting a too high a threshold of around $t h r=0.95$ for a link with a high variance in the delivery rate vs power curve could cause ConTPC to decide that the flat area is quite narrow. This could effectively disable ConTPC on links with high variance of the delivery rate vs power characteristics. However, threshold val- 


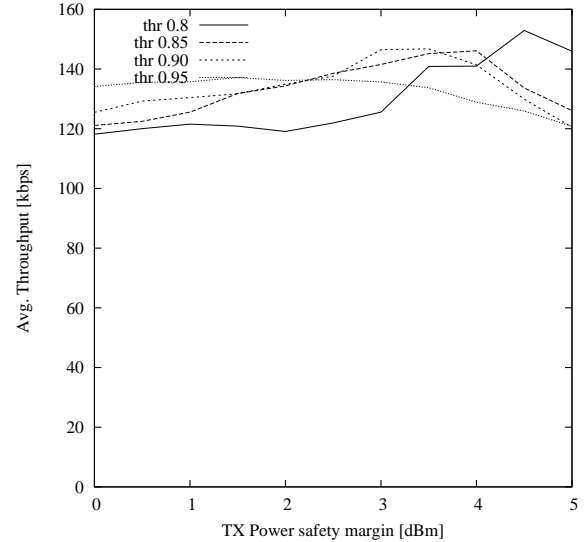

Figure 15: Evaluation of importance of $P_{\text {safety }}$ and $t h r$ parameters

ues of around 0.8 ensure that ConTPC correctly finds the boundary of the flat area despite some variability. While a large $P_{\text {safety }}($ above $3 \mathrm{dBm}$ ) ensures that only good quality links reduce their power. Consequently, by being conservative this allows for the efficient operation of ConTPC.

\section{CONCLUSIONS}

In this paper we have presented the ConTPC mechanism which is a conservative transmit power control scheme. It does not seek to reduce the transmit power for all the networks links because such an approach usually penalises low quality links. Instead, it only reduces the transmit power for links on which such a reduction does not affect the delivery rate.

We have demonstrated that the performance of ConTPC is topology dependent. The highest throughput gain can be observed in nonuniform topologies such as the disjoint node pairs case, when multiple transmissions may occur simultaneously. However, in a typical indoor deployment, as for example in case of our test bed, such a scheme continues to outperform the fixed power levels case, resulting in a $15 \%$ throughput increase when compared with fixed power operating at its maximum value.

We have also shown that ConTPC not only increases the overall throughput but also increases the fairness by reducing contention. Moreover, it is possible to control the overhead of ConTPC while maintaining its high throughput gain.

\section{ACKNOWLEDGEMENT}

The authors acknowledge the support of Enterprise Ireland (under the Commercialisation Fund 2007) and Science Foundation Ireland (under the grant 03/IN3/I396).

\section{REFERENCES}

(2001). A power controlled multiple access protocol for wireless packet networks. INFOCOM 2001. Twentieth Annual Joint Conference of the IEEE Computer and Communications Societies, 1(4):219 - 228. J.P. Monks and V. Bharghavan and W.-M.W. Hwu.

802.11h IEEE Standard for Information technology Telecommunications and information exchange between systems - Local and metropolitan area networks - Specific requirements. Part 11: Wireless LAN Medium Access Control (MAC) and Physical Layer (PHY) specifications, Amendment 5: Spectrum and Transmit Power Management Extensions in the $5 \mathrm{GHz}$ band in Europe.

Akella, A., Judd, G., Seshan, S., and Steenkiste, P. (2005). Self-management in chaotic wireless deployments. In MobiCom 105: Proceedings of the 11th annual international conference on Mobile computing and networking, pages 185-199.

Broustis, I., Eriksson, J., Krishnamurthy, S. V., and Faloutsos, M. (2007). Implications of power control in wireless networks: A quantitative study. In Proceedings of Passive and Active Measurement Conference (PAM).

Gomez, J., Campbell, A., Naghshineh, M., and Bisdikian, C. (2003). PARO: Supporting Dynamic Power Controlled Routing in Wireless Ad Hoc Networks. ACM/Kluwer Journal on Wireless Networks (WINET), 9(5):443-460.

Jain, K., Padhye, J., Padmanabhan, V. N., , and Qiu, L. (2003). Impact of interference on multi-hop wireless network performance. ACM MobiCom.

Kawadia, V. and Kumar, P. (2005). Principles and protocols for power control in wireless ad hoc networks. IEEE Journal on Selected Areas in Communications, 23(1):76-88

Kohler, E., Morris, R., Chen, B., Jannotti, J., and Kaashoek, M. F. (2000). The Click modular router. ACM Transactions on Computer Systems, 4(18).

Kowalik, K., Bykowski, M., Keegan, B., and Davis, M. (2008). Practical Issues of Power Control in IEEE 802.11 Wireless Devices. In Proceedings of 15th International Conference on Telecommunications ICT'08, St. Petersburg, Russia.

Muqattash, A. and Krunz, M. (2003). Power controlled dual channel (PCDC) medium access protocol for wireless ad hoc networks. INFOCOM 2003, pages 470-480.

Muqattash, A. and Krunz, M. (2005). POWMAC: A SingleChannel Power-Control Protocol for Throughput Enhancement in Wireless Ad Hoc Networks. IEEE Journal on Selected Areas in Communications (JSAC) - 
Special Issue on Advances in Military Wireless Communications, 23(5):1067-1084.

Navda, V., Kokku, R., Ganguly, S., and Das, S. (2007). Per-client Power Management for Spatial Reuse in WLANs. Technical Report, Computer Science Department, Stony Brook University, NY.

Padhye, J., Agarwal, S., Padmanabhan, V., Qiu, L., Rao, A., and Zill, B. (2005). Estimation of Link Interference in Static Multi-hop Wireless Networks. Internet Measurements Conference.

Ramanathan, R. and Hain, R. (2000). Topology control of multihop wireless networks using transmit power adjustment. In INFOCOM, pages 404-413.

Son, D., Krishnamachari, B., and Heidemann, J. (2004). Experimental study of the effects of transmission power control and blacklisting in wireless sensor networks. In Proceedings of the First IEEE Conference on Sensor and Adhoc Communication and Networks, pages 289-298. 